\section{ADVERSE REACTIONS IN STATIN THERAPY AT YOUNGER AND CHILDREN OLDER THEN TWELVE YEARS}

A. Bajraktarevic ${ }^{1}$, M. Miokovic ${ }^{1}$, S. Trninic ${ }^{1}$, S. Putica ${ }^{1}$, Z. Margeta ${ }^{1}$, D. Abduzaimovic ${ }^{2}$, J. Ceman Saric ${ }^{3}$, H. Niksic ${ }^{4}$, Z. Begic ${ }^{5}$, A. Selimovic ${ }^{5}$, S. Majstorovic ${ }^{5}$

${ }^{1}$ Pediatrics Department, Public Health Institution of Canton Sarajevo-Pediatrics Department, ${ }^{2}$ Biochemistry Department, Private Biochemistry Laboratory Tesanj, ${ }^{3}$ Biochemistry Department, Clinical Medical Center Sarajevo, ${ }^{4}$ Clinical Pharmacology Department, Pharmaceutical Faculty Sarajevo, ${ }^{5}$ General Pediatrics Department, Pediatrics Clinic Sarajevo, Sarajevo, Bosnia-Herzegovina

Background: Obesity in childhood bodes ill for future cardiovascular risk. HMG-CoA reductase is an enzyme in the cholesterol biosynthetic pathway that catalyses the conversion of HMG-CoA to mevalonic acid. Statins are a class of drugs that lower the level of cholesterol in the blood by reducing the production of cholesterol by the liver.

Aims: The most controversial change appears to have been the inclusion of statins as potential firstline pharmacologic agents in older children.

Methods: High-risk children, include those who are obese or overweight and who also have high blood pressure, or diabetes, or a positive family history of either high cholesterol or early heart disease. The safety of statins in children and adolescents is based on trials that have ranged in duration from six months to one year.

Results: Inhibition of this enzyme in the liver results in decreased cholesterol synthesis as well as increased synthesis of LDL receptors, resulting in an increased clearance of low-density lipoprotein (LDL) from the bloodstream. The first results can be seen after one week of use and the effect is maximal after four to six weeks. No serious laboratory adverse events were reported during follow-up, and statin treatment had no untoward effects on sexual maturation.

Conclusion: The recommendation to use statins in childhood seems to have hit a collective nerve, perhaps awakening us to the fuller implications of the obesity epidemic. Statins exhibit action beyond lipid-lowering activity in the prevention of atherosclerosis.
Keywords: Obesity, Younger , Children, Statins, Therapy, Contraindications.

541

\section{THE DIAGNOSIS AND TREATMENT PARTICULARITIES IN A CASE WITH JUVENILE IDIOPATHIC ARTHRITIS}

\author{
S.I. Iurian ${ }^{1,2}$, M.L. Neamtu ${ }^{1,2}$, B.M. Neamtu ${ }^{1,2}$, \\ C. Soroiu², G. Gradinariu² \\ ${ }^{1}$ Lucian Blaga University, ${ }^{2}$ Pediatric Clinic, \\ Sibiu, Romania
}

Background and aims: To emphasize the diagnosis peculiarities and treatment difficulties in a case with joint pains.

Methods: The authors present a 15 year-old girl admitted for chronic limping. Personal history: morning stiffness and progressive walking difficulties due to arthralgia during last 12 months, progressive regression in motor skills. The girl has followed a chronic therapy with retard penicillines because of persistent high ASLO titer (rheumatic fever suspicion). Clinical exam: impaired nutritional status (- $3 \mathrm{SD}$ ), symmetric joint involvement: pain and swelling for proximal interphalangeal hand joints, metacarpophalangeal joints, wrists, knees, ankles and temporomandibular joints, boutonniere deformities and ankylosis for hand fingers, hands and legs muscles atrophy.

Results: Laboratory investigations: elevated inflammatory markers, negative rheumatoid factor, positive antinuclear antibodies, high ASLO titer, hypergammaglobulinemia. The imagistic approach: periarticular osteoporosis (wrist radiographs), narrowed joint spaces and erosions. Synovial biopsy has revealed suggestive features for juvenile idiopathic arthritis (JIA). According to diagnosis, the treatment has included physical therapy, anti-inflammatory drugs and methotrexate. Even under methotrexate therapy, the joint function has deteriorated with hand fingers progressive ankylosis. The biologic therapy (etanerceptum) has been initiated with improvement of joint mobility, synovial inflammation and osteoporosis, too.

Conclusions: The patient was misdiagnosed as rheumatic fever due to high ASLO titers that can be explained by polyclonal and non-selective B lymphocytes activation. In this case, ASLO titer was correlated with disease's activity. The case peculiarity: it's important to consider the JIA diagnosis for cases with persistent high ASLO titers and unresponsiveness to penicillin therapy. 